International Journal of Wireless \& Mobile Networks (IJWMN) Vol. 4, No. 4, August 2012

\title{
LOAD BALANCING BASED APPROACH TO IMPROVE LIFETIME OF WIRELESS SENSOR NETWORK
}

\author{
Dipak Wajgi ${ }^{1}$ and Dr. Nileshsingh V. Thakur ${ }^{2}$ \\ ${ }^{1}$ Department of Computer Science and Engineering, Shri. Ramdeobaba College of \\ Engineering and Management, Nagpur, India \\ wajgi@rediffmail.com \\ ${ }^{2}$ Department of Computer Science and Engineering, Shri. Ramdeobaba College of \\ Engineering and Management, Nagpur, India \\ thakurnisviserediffmail.com
}

\begin{abstract}
In wireless sensor network, clustering is used as an effective technique to achieve scalability, selforganization, power saving, channel access, routing etc.[3]. Lifetime of sensor nodes determines the lifetime of the network and is crucial for the sensing capability.[2]. Clustering is the key technique used to extend the lifetime of a sensor network. Clustering can be used for load balancing to extend the lifetime of a sensor network by reducing energy consumption. Load balancing using clustering can also increase network scalability. Wireless sensor network with the nodes with different energy levels can prolong the network lifetime of the network and also its reliability. In this paper we propose a clustering technique which will balance the load among the cluster by using some backup nodes. The backup high energy and high processing power nodes replace the cluster head after the cluster reaches to its threshold limit. This approach will increase the network lifetime and will provide high throughput.
\end{abstract}

\section{KEYWORDS}

wireless sensor network, clustering, reliability, scalability

\section{INTRODUCTION}

Information gathering is a fast growing and challenging field in today's world of computing. Sensors provide a cheap and easy solution to these applications especially in the inhospitable and low-maintenance areas where conventional approaches prove to be very costly. Sensors are tiny devices that are capable of gathering physical information like heat, light or motion of an object or environment. Sensors are deployed in an ad-hoc manner in the area of interest to monitor events and gather data about the environment. Networking of these unattended sensors is expected to have significant impact on the efficiency of many military and civil applications, such as combat field surveillance, security and disaster management. Sensors in such systems are typically disposable and expected to last until their energy drains. Therefore, energy is a very scarce resource for such sensor systems and has to be managed wisely in order to extend the life of the sensors for the duration of a particular mission. Typically sensor networks follow the model of a base station or command node, where sensors relay streams of data to the command node either periodically or based on events. The command node can be statically located in the vicinity of the sensors or it can be mobile so that it can move around the sensors and collect data. In either case, the command node cannot be reached efficiently by all the sensors in the system. The nodes that are located far away from the command node will consume more energy to transmit data then other nodes and therefore will die sooner[6]. 
International Journal of Wireless \& Mobile Networks (IJWMN) Vol. 4, No. 4, August 2012

A wireless sensor network is typically consisting of a potentially large number of resource constrained sensor nodes and few relatively powerful control nodes. Each sensor node has a battery and a low-end processor, a limited amount of memory, and a low power communication module capable of short range wireless communication [3]. As sensor nodes have very limited battery power and they are randomly deployed it is impossible to recharge the dead battery. So the battery power in WSN is considered as scarce resource and should be efficiently used. Sensor node consumes battery in sensing data, receiving data, sending data and processing data [1].

Generally a sensor node does not have sufficient power to send the data or message directly to the base station. Hence, along with sensing the data the sensor node act as a router to propagate the data of its neighbour.

In large sensor network, the sensor nodes can be grouped into small clusters. Each cluster has a cluster head to coordinate the nodes in the cluster. Cluster structure can prolong the lifetime of the sensor network by making the cluster head aggregate data from the nodes in the cluster and send it to the base station. A randomly deployed sensor network requires a cluster formation protocol to partition the network into clusters. The cluster heads should also be selected. There are two approaches used in this process the leader first and the cluster first approach. In the leader first approach the cluster head is selected first and then cluster is formed. In the cluster first approach the cluster is formed first and then the cluster head is selected [3].

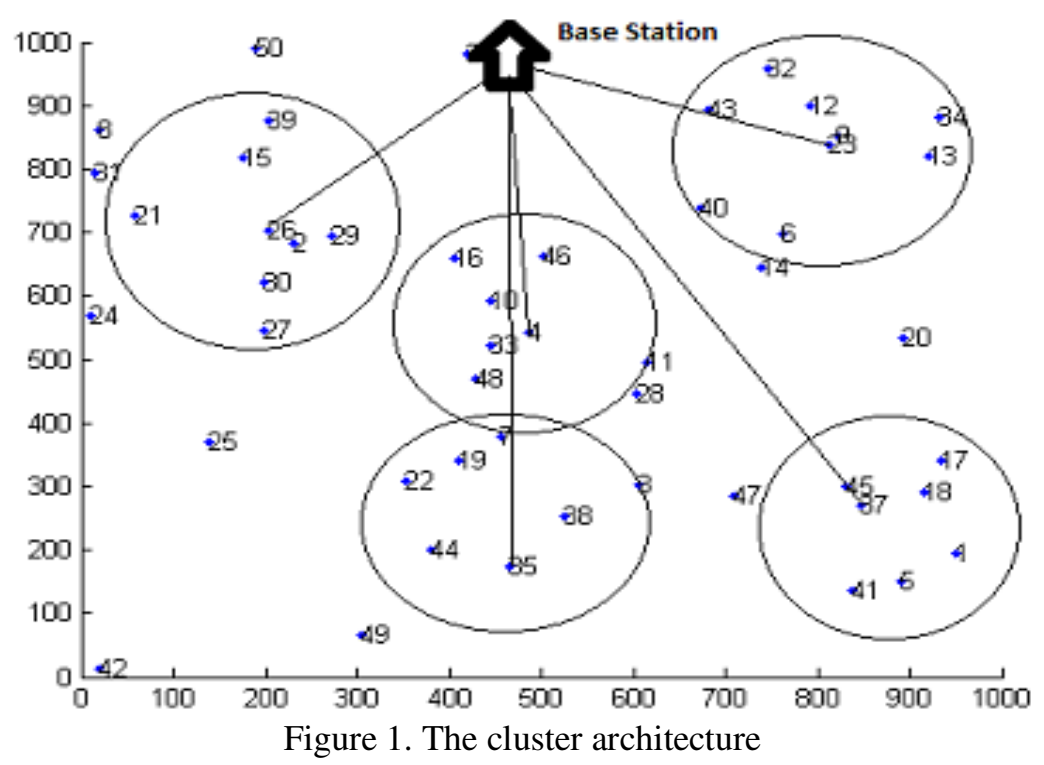

Figure1 represent the cluster architecture of the sensor nodes. Initially the sensor nodes are randomly deployed. The nodes are heterogeneous in nature with the different energy levels. The nodes with the higher energy are found in the region and are made cluster head. All the cluster heads defines its range and form the cluster. The cluster member nodes send the sensed data to the cluster heads and cluster head then sends that data to the base station. In most of the cases it is assumed that the cluster head is sending the data to the base station directly.

Clustering has numerous advantages like it reduces the size of the routing table, conserve communication bandwidth, prolong network lifetime, decrease the redundancy of data packets, reduces the rate of energy consumption etc[4]. 
International Journal of Wireless \& Mobile Networks (IJWMN) Vol. 4, No. 4, August 2012

Generally it is assumed that the nodes in wireless sensor networks are homogeneous, but in reality, homogeneous sensor networks hardly exist. Even homogeneous sensors have different capabilities like different levels of initial energy, depletion rate, etc. In heterogeneous sensor networks, typically, a large number of inexpensive nodes perform sensing, while a few nodes having comparatively more energy perform data filtering, fusion and transport. This leads to the research on heterogeneous networks where two or more types of nodes are considered. Heterogeneity in wireless sensor networks can be used to prolong the life time and reliability of the network. Heterogeneous sensor networks are very popular [4].

\subsection{Heterogeneous Model for Wireless Sensor Networks}

Different Heterogeneous Model for Wireless Sensor Networks is suggested based on various resources. There are three common types of resource heterogeneity in sensor nodes: computational heterogeneity, link heterogeneity, and energy heterogeneity.

Computational heterogeneity means that the heterogeneous node has a more powerful microprocessor, and more memory, than the normal node. With the powerful computational resources, the heterogeneous nodes can provide complex data processing and longer term storage.

Link heterogeneity means that the heterogeneous node has high bandwidth and long distance network transceiver than the normal node. Link heterogeneity can provide a more reliable data transmission.

Energy heterogeneity means that the heterogeneous node is line powered, or its battery is replaceable.

Among above three types of resource heterogeneity, the most important heterogeneity is the energy heterogeneity because both computational heterogeneity and link heterogeneity consumes more energy resource.

The remainder of the paper is organised as follows. In section 2, review of literature is discussed. In section 3, design philosophy is discussed. In section 4, load balancing approach is proposed and explained. Simulation results are presented in section 5. Section 6 concludes the paper with conclusion and future scope.

\section{RELATED WORK}

Clustering can be used for balancing the load in the wireless sensor networks[6,8,9,10,12,13,14,18,19,20,21,22,24,25,]. In a cluster based load balancing [6], the maximum transmission power of the nodes is used to become the cluster member. Cluster membership depends on the communication cost. The proposed approach does not consider the backup recovery. A load balanced clustering approach [8], uses comprehensive weight value composed of distance between the head and the member and the residual energy to improve cluster member choice. It also uses optimization threshold value to avoid load imbalance. The algorithm considers load equalization for creating balanced cluster. A multi-hop clustering algorithm for load balancing in wireless sensor networks [9], uses layered approach for intra cluster and inter cluster communication. The algorithm consider homogeneous network. Reconfiguration of cluster head for load balancing in wireless sensor networks [10], increases the network lifetime by fairly distributing the cluster heads. Reconfiguration of the cluster is done based of the number of general nodes in the cluster \& the number of cluster heads within the cluster head's transmission range. The algorithm provides effective data aggregation. A 
International Journal of Wireless \& Mobile Networks (IJWMN) Vol. 4, No. 4, August 2012

novel load balancing scheduling algorithm for wireless sensor networks [12], uses optimal scheduling algorithm for packet forwarding which determines the time slot for sending the packets for the nodes. The algorithm provides uniform packet loss probability for all the nodes. The algorithm uses balanced cost objective function for optimum scheduling. Secure load balancing via hierarchical data aggregation in heterogeneous wireless sensor networks [13], protocol introduces pseudo sink in order to improve data accuracy and bandwidth utilization of wireless sensor network to increase network lifetime. A load balanced algorithm in wireless sensor networks based on pruning mechanism [14], handles the hot point problems which uses pruning mechanism in the cluster to balance the load in the network. Evaluation function in the algorithm is based on pruning mechanism and uses nodes location, residual energy and count of cluster nodes as its parameter to find its cost. In load balancing in energy efficient connected coverage wireless sensor networks [18], the algorithm consider sensing coverage \& network connectivity by dividing the sensor network nodes into subsets. It turns on some extra nodes in each subset to ensure network connectivity. The problem with this approach is to find the existence of critical nodes. These nodes may be on all the time and the network will be partitioned if these nodes die. A Threshold Based algorithm for Power aware Load Balancing in Sensor Networks [19], provides possible in-network method for adaptive distributed control of energy consumption. Other methodologies like market based algorithm or game theoretic algorithm can be used. The algorithm assumes complete connectivity. A Load Balanced Clustering Algorithm for Wireless Sensor Networks [20], has Proposed the load balancing algorithm for cluster heads in wireless sensor networks by considering the traffic load as the key parameter. It is assumed that the traffic loads contributed by all the sensor nodes are same, which is the special case of this algorithm. In general case the algorithm is NP hard. It uses centralized approach and assumes that each node is aware of the network. Clustering and Load Balancing in Hybrid Sensor Network with mobile Cluster Nodes[21], has proposed an algorithm that consider the problem of positioning mobile cluster heads and balancing traffic load in hybrid sensor network which consists of static and mobile nodes. It is stated that the location of the cluster head can affect network lifetime significantly. Network load can be balanced and lifetime can be prolonged by moving cluster head to better location. A Load balanced Clustering Algorithm for Heterogeneous Wireless Sensor Networks[22], has Proposed the load balanced group clustering to balance the battery power in wireless sensor network by implementing dynamic route calculation according to the condition of energy distribution in the network. It makes use of heterogeneous energy to realize load balance. Fuzzy Based Approach for Load Balanced Distributing database on Sensor Networks [24], has proposed fuzzy based approach for load balanced distributing database on sensor network that prolong the network lifetime. In this algorithm vertical partitioning algorithm for distributing database on sensors is used. In this approach, first clusters are formed and then distribute partitions on clusters. In an Energy Aware Dynamic Clustering Algorithm for Load Balancing in Wireless sensor network [25], a novel dynamic clustering algorithm for load balanced routing is proposed which is based upon route efficiency. The algorithm uses pattern, traffic load \& energy dissipation rate of each node on the route to calculate the node and route efficiency. Energy Efficient Communication Protocol for Wireless Sensor Networks [28], uses low energy adaptive clustering hierarchy that utilizes randomized rotation of local cluster base station to evenly distribute the energy load among sensors of the network. This protocol provides scalability and robustness for dynamic networks. It incorporates data fusion into routing protocol to reduce the amount of information transmitted to the base station. It uses minimum distance from the cluster head to the other nodes as the parameter for the cluster formation. The algorithm is also organized in such a manner that data fusion can be used to reduce the amount of data transmission. The decision of whether a node elevates to cluster head is made dynamically at each interval. The elevation decision is made solely by each node independent of other nodes to minimize overhead in cluster head establishment. This decision is a function of the percentage of optimal on application cluster heads in a network (determined a priory on application), in combination with how often and the 
International Journal of Wireless \& Mobile Networks (IJWMN) Vol. 4, No. 4, August 2012

last time a given node has been a cluster head in the past. In this algorithm each node calculates the minimum transmission energy to communicate with its cluster head and only transmits with that power level.

Routing can also be used to balance the load in the wireless sensor network [7, 11, 15, 16, 17, 23, 26, 27]. Multipath Routing Algorithm for wireless sensor network [7], finds the node disjoint routing path similar to mazing search. It reduces the energy consumption and congestion. It introduces Multi path selection strategy to balance the load in the network. The bandwidth is utilized efficiently for transmitting audio and video packets. Energy Aware Intra Cluster Routing for wireless sensor network [11], performs adaptive routing where the distance from base station is taken into consideration. Multihop Routing Based on Optimization of the number of cluster heads in wireless sensor network [15], has proposed the equation for the number of packets to send and relay to calculate the energy consumption of sensor nodes. Change in the number of cluster heads affects the consumed energy of sensor nodes. Overlapping Multihop Clustering for wireless sensor network [16], has proposed Randomized, distributed multihop clustering protocol for solving overlapping clustering problems. It considers average overlapping degree. It is scalable. Cluster formation terminates in constant time regardless for network size. A Novel Cluster Based Routing Protocol with Extending Lifetime for Wireless Sensor Networks [17], has self configuration and hierarchical routing properties. It construct cluster based on radio radius and number of cluster members. Clusters in the network are equally distributed. Sensor nodes perform voting for selecting cluster head. An Energy Aware Cluster Based Routing Algorithm for Wireless Sensor Networks [23], has proposed the algorithm for wireless sensor network to maximize network's lifetime. It selects some nodes as cluster heads to construct voronoi diagram and rotate the cluster head to balance the load in each other. A cluster Based Energy Efficient Location Routing Protocol in Wireless Sensor Networks [26], uses hierarchical structured method, multihop and location based nodes. It works well for small networks. Cluster head is selected based on residual energy and minimum distance from the base station. In a Novel Cluster Based Routing Protocol in Wireless Sensor Networks [27], a cluster based routing protocol for prolonging the sensor network lifetime is proposed. The algorithm achieves a good performance in terms of lifetime by balancing the energy load among all the nodes. Cluster Based Routing Protocol achieves a good performance in terms of lifetime by balancing the energy load among all the nodes. In this protocol first the clusters are formed then the spanning tree is constructed for sending aggregated data to the base station which can better handle the heterogeneous energy capacities.

\section{DeSIGN PHILOSOPHY}

Wireless Sensor Networks present vast challenges in terms of implementation. Clustering algorithms play a vital role in achieving the targeted design goals for a given implementation. There are several key attributes that must be considered in wireless sensor networks [5].

Selection of Cluster heads and Clusters: The clustering concept offers tremendous benefits for wireless sensor networks. However when designing for a particular application, designers must carefully examine the formation of clusters in the network. Depending on the application, certain requirements for the number of nodes in a cluster or its physical size may play an important role in its operation. This prerequisite may have an impact on how cluster heads are selected.

Data Aggregation: One major advantage of wireless sensor networks is the ability for data aggregation to occur in the network. In a densely populated network there are often multiple nodes sensing similar information. Data aggregation allows the differentiation between sensed data and useful data. Network processing makes this process possible and now it is fundamental 
International Journal of Wireless \& Mobile Networks (IJWMN) Vol. 4, No. 4, August 2012

in many sensor network schemes, as the power required for processing tasks is substantially less than communication tasks. As such, the amount of data transferred in network should be minimized. Many clustering schemes provide data aggregation capabilities, and as such, the requirement for data aggregation should be carefully considered when selecting a clustering approach.

Repair Mechanisms: Due to the nature of Wireless Sensor Networks, they are often prone to node mobility, node death and interference. All of these situations can result in link failure. When looking at clustering schemes, it is important to look at the mechanisms in place for link recovery and reliable data communication.

\section{Proposed Approach}

The proposed approach assumes heterogeneous network with the sensor nodes having different energy levels and processing power. Some high computing nodes are deployed nearby each other. All the nodes with high initial energy level and processing power are selected. Some nodes from the set are selected as cluster head $(\mathrm{CH})$ according to their location. Each $\mathrm{CH}$ defines its communication range in terms of power level to form cluster. Some nodes with comparable energy and processing power in the $\mathrm{CH}$ range are asked to go to sleep and information about those nodes is maintained with the $\mathrm{CH}$. Each $\mathrm{CH}$ sends a hello request message to all the nodes within its communication range to become the cluster member. This process will be repeated for all the $\mathrm{CH}$. All the cluster members will send the sensed data to the $\mathrm{CH}$. The $\mathrm{CH}$ will send the aggregated data to the Base Station directly or by using some intermediate $\mathrm{CH}$.

When the energy level of the $\mathrm{CH}$ will reach to the threshold value $\mathrm{TL}$, the $\mathrm{CH}$ will activate one of the sleeping nodes and will make it $\mathrm{CH}$. This information about the new $\mathrm{CH}$ will be sent to all the cluster member and other $\mathrm{CH}$ also. The old $\mathrm{CH}$ will become the general sensor node.

\subsection{ALgORithm}

The algorithm is divided into four phases

\section{Initialization Phase}

1.1 Select the $\mathrm{CH}$ according to the capabilities of the nodes.

1.2 Select the desired number of $\mathrm{CH}$ according to their location.

1.3 Define the range of $\mathrm{CH}$.

1.4 $\mathrm{CH}$ sends membership request message to all the nodes in its range and request to reply with their current energy status.

1.5 The nodes with high residual energy and processing power will be identified and they are made to sleep. They become the backup nodes.

1.6 The nodes which are not in the range of cluster head, will try to join the cluster by sending the message to the nearest cluster member.

\section{Steady State Phase}

2.1 The cluster members sends the sensed data to the $\mathrm{CH}$ in the allotted time using TDMA schedule.

2.2 The non cluster members will send the sensed data to the cluster head through the intermediate cluster member.

\section{Final Phase}

3.1 $\mathrm{CH}$ will aggregate the data from all the nodes in its cluster.

3.2 $\mathrm{CH}$ will transmit the data to the base station.

\section{Cluster Reconfiguration Phase}

4.1 If the $\mathrm{CH}$ residual energy reaches to the threshold value, the $\mathrm{CH}$ will activate the backup node. 
International Journal of Wireless \& Mobile Networks (IJWMN) Vol. 4, No. 4, August 2012

4.2 The $\mathrm{CH}$ will relegate its responsibility to the backup node and will make the node the cluster head.

4.3 The $\mathrm{CH}$ will transmit the new $\mathrm{CH}$ information to all other nodes in the cluster.

4.4 The $\mathrm{CH}$ will transmit the new $\mathrm{CH}$ information to all other $\mathrm{CH}$ also.

4.5 The old $\mathrm{CH}$ will become the general node.

\section{EXPERIMENTAL SETUP AND RESUltS}

The sensor nodes are assumed to be distributed randomly.

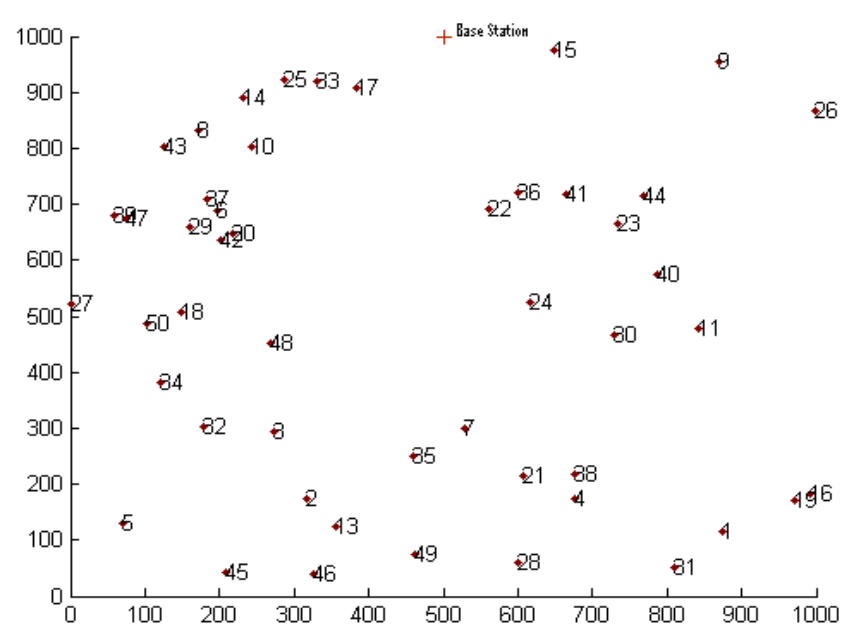

Figure 2. The random distribution of the sensor nodes.

Figure 2 represent the random distribution of the sensor nodes in MATLAB. The flat area is considered for the distribution of the sensor nodes. The position of the base station is also fixed. In this random distribution, the areas are found where the density of the sensor nodes is more. Accordingly the nodes with the higher energy and high processing power will be deployed. While deploying the high energy and high processing power nodes the care is taken that the backup nodes are also deployed nearby the high energy and high processing power nodes. Here in this case, consider these nodes are 37 and 6,2 and 13,4 and 38 and 23 and 40. We are creating the pairs of the nodes so that if one node fails or reaches to the threshold energy value the backup node will take the responsibility of that node.

\subsection{Clustering Process}

Cluster formation process is shown in the Figure 3. The high energy and high processing power nodes which are the cluster heads defines their range of communication in terms of distance. In the above figure the nodes 37, 2,4 and 23 are acting as cluster heads. Then they send the membership request message to the sensor nodes in their communication range. The sensor nodes are requested to send the acceptance message along with their energy status to the requesting cluster head. After receiving the acceptance message and the energy information, the cluster head finds the node with the energy and power comparable to it. If found, it allows the node to go to sleep. This node acts as a backup node for the cluster head. In the above figure the node 6 is acting as a backup nodes for node 37, node 13 is acting as a backup nodes for node 2 , node 38 is acting as a backup nodes for node 4 , node 40 is acting as a backup nodes for node 
International Journal of Wireless \& Mobile Networks (IJWMN) Vol. 4, No. 4, August 2012

23. In the sleeping mode also the sensor node is dissipating the energy but with very less rate. All the cluster heads forms their clusters along with the backup nodes.

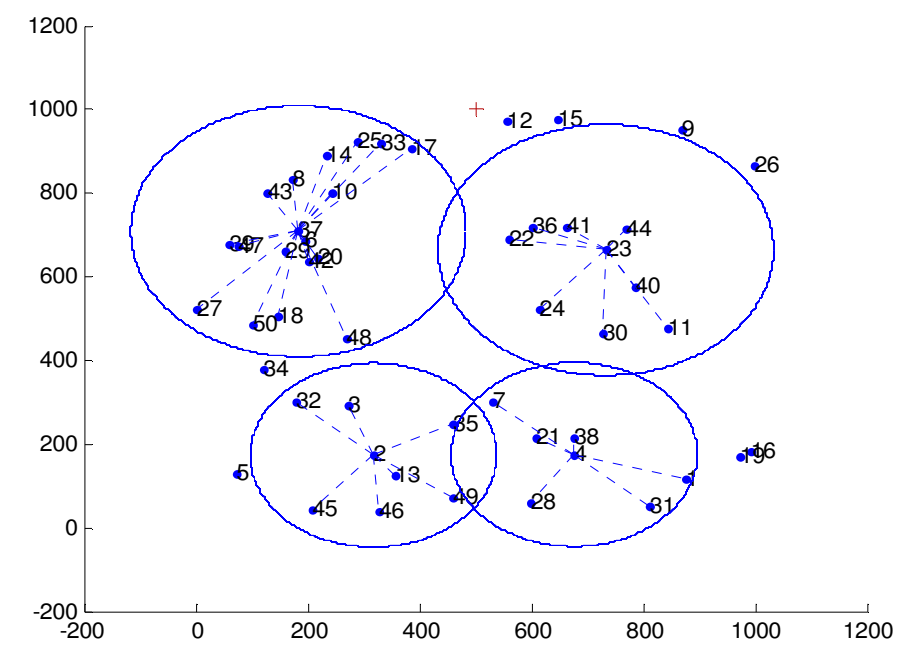

Figure 3. Cluster formation

There are some nodes which are not the member of any cluster. These nodes search for the minimum distance node which is the member of any one of the cluster. In the figure 4, node 12 is attached to the cluster of cluster head 37 through the node 17 and node 15 is attached to the cluster of cluster head 23 through node 41 . Like this all the remaining nodes are attached to the cluster through the intermediate node which is the cluster member of the particular cluster.

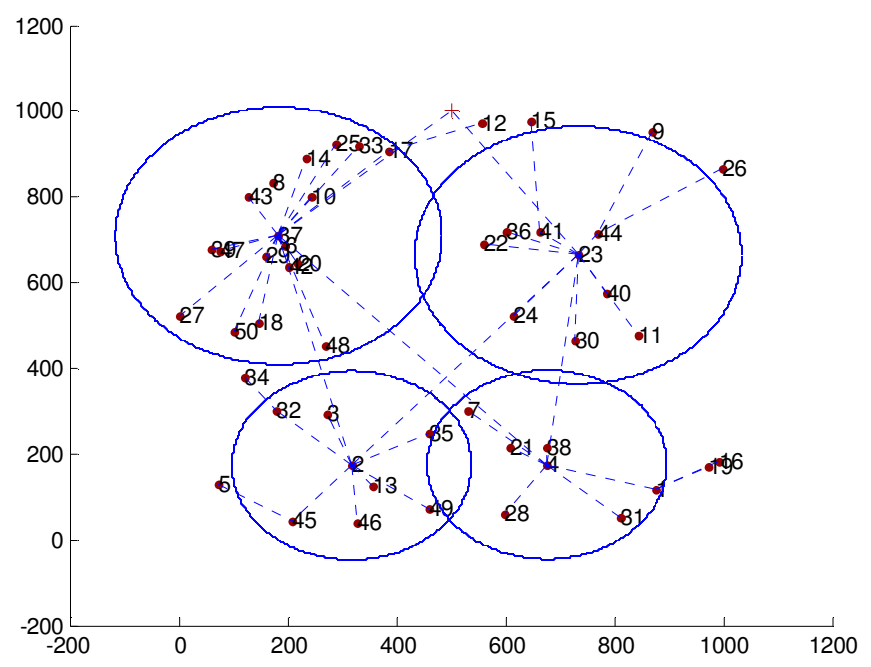

Figure 4. Connection among the cluster heads and non cluster member nodes.

\subsection{ReCONFIGURATION OF Clusters}

The cluster reconfiguration process is shown in Figure 5. After some time period which is modelled as rounds, the energy level of the cluster head reaches to the threshold value. This threshold value is already set which is the indicator that the cluster head can no longer handle 
the responsibility of the head and should hand over the responsibility to the backup node. The cluster head awake the backup node which is in the sleep node and ask it to send the hello message to the nodes in the range. At the same time it sends the message to all the member nodes informing about the new cluster head. If any member node, not getting the hello message from the new cluster head, it means that the node is not within the range of the new cluster head and should find the new cluster. This process is followed by all the cluster heads. In this process the membership of the nodes can be changed i.e. the node which is the member of one cluster may become the member of another cluster after reconfiguration and the nodes which is not the direct member of any cluster may become the direct member of the cluster.

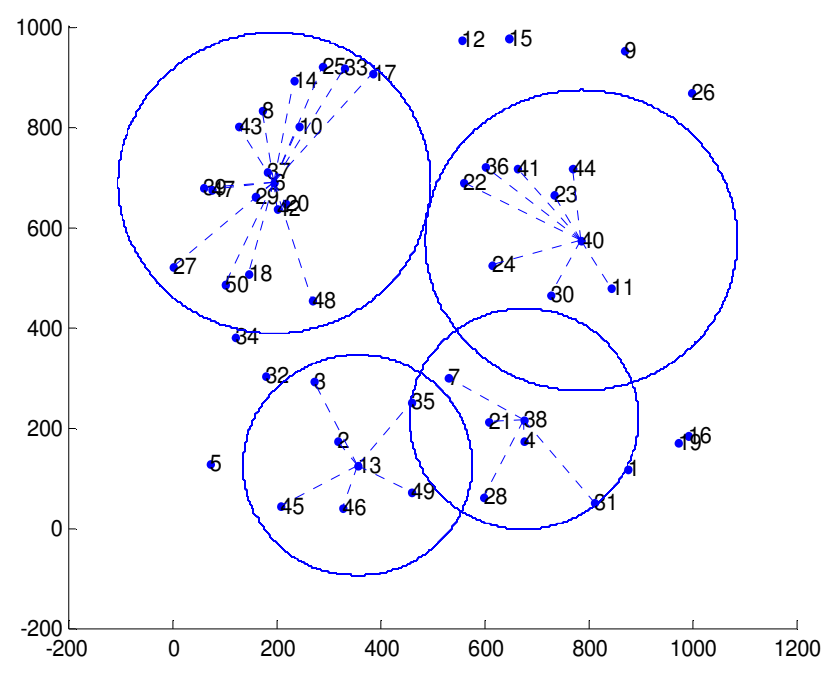

Figure 5. Reconfiguration of cluster

After completing this reconfiguration phase, some nodes, which are not the members of any of the cluster, try to find the nearest cluster member node. Again, the same criterion of finding the minimum distance cluster member is applied to find the appropriate cluster. The Figure 6 shows the connection among the new cluster heads and the remaining nodes. So these remaining nodes are indirectly attached to the new clusters.

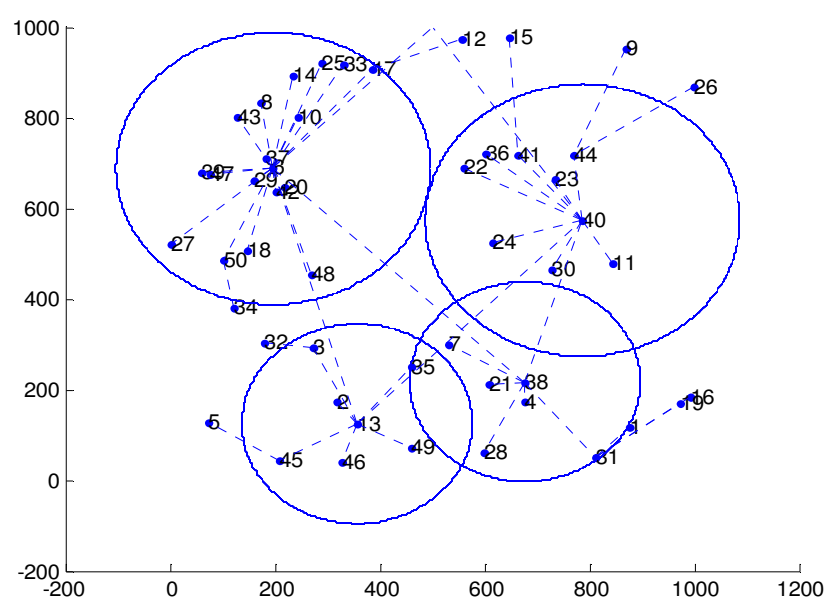

Figure 6. Connection among the new cluster heads and non cluster member nodes. 


\subsection{Performance Graph}

The performance of the proposed algorithm is plotted in the graph showing the number of the nodes against the number of rounds in the Figure 7 . The round starts with the initialization phase of creating the clusters. After the clusters are formed the sensor nodes are aggregating the data and sending it to the cluster heads. Before this the base station is giving some instructions to the cluster heads for collecting some information in the particular area. The cluster heads pass on this information to the cluster member nodes. In turn, the member nodes collect the information and send it to the cluster head and then cluster head sends the collected information to the base station for the further processing. The energy of the sensor node is consumed while sensing the data, performing some operations on data. But most of the energy of the sensor nodes is consumed during communication. The dissipation of the energy during communication is depending on the distance between the sensor nodes. If the distance is longer then more energy gets dissipated and if the distance is small then less energy is dissipated.

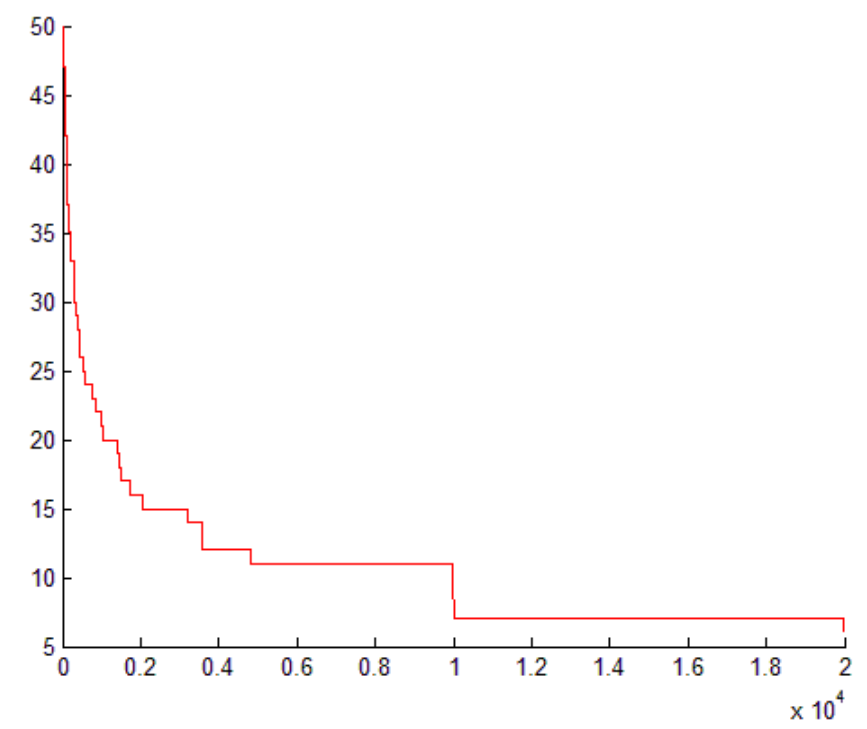

Figure 7. Performance graph showing the number of nodes on y-axis and the rounds on the $\mathrm{x}$-axis.

If all the sensor nodes are sending the data to the base station directly, then the lifetime of the sensor network gets reduced since the base station is located far away from the senor nodes and the energy dissipated for this long haul communication is very much high. This is one of the reasons behind going for clustering techniques. The sensor node communication is limited within the cluster. So the energy dissipated will be less. The cluster heads are responsible for communication with the base station. Being the high energy and high processing power nodes, they perform the long haul communication and thus prolong the network lifetime.

If all the cluster heads are sending the data to the base station then some of the cluster heads may die sooner since they are at long distance. So some routing mechanism is also employed to prolong the network lifetime. The long distance cluster heads send the data to the base station via some intermediate cluster heads. 
International Journal of Wireless \& Mobile Networks (IJWMN) Vol. 4, No. 4, August 2012

If all the long distance cluster heads are sending the data to the cluster heads which are close to the base station, then there are chances that the cluster heads close to the base station will die sooner, since along with the data aggregation and sending their own data they have to aggregate the data from the other cluster heads and send it to the base station. To avoid this, routing in used where the distant cluster heads will not send the data to the same cluster head near the base station in every round but they will send the data every alternate round.

The nodes which are not the direct member of the cluster send the data to the member node of the cluster and in turn the member node along with its own data sends it to the cluster head. Energy dissipation of the member nodes and non-member nodes depends on the distance from the cluster head. If the distance is more, then more energy is dissipated otherwise less energy is dissipated.

In the Figure 7, the graph is plotted for the number of live nodes against the number of rounds. The time from the initialization phase to the first node death defines the network lifetime. If the results are compared with the original LEACH algorithm, the first clustering algorithm, with the same experimental setup, then the results are better. Even with the first node death, unless and until all the cluster head die the network is performing its job.

\section{CONClusion ANd Future SCOPE}

In this paper, an approach for load balancing in the wireless sensor network is proposed. Algorithms for cluster head selection, cluster formation, intra cluster communication and inter cluster communication in wireless sensor network are proposed. The performance of the algorithm is compared with the original LEACH algorithm with respect to the number of rounds and the dead nodes using the parameter like energy dissipation in each round per node. The results demonstrate that the proposed approach is effective in prolonging the network lifetime.

In future, experiments are planned to be extended for parameters and scenarios like coverage, fault tolerance, impact of aggregation and mobility of nodes.

The proposed approach is implemented by using MATLAB. In future the tools like NS-2 and OMNET++ can be used for implementation.

\section{References}

[1] Babar Nazir, Halabi Hasbullah,” Energy Balanced Clustering in Wireless Sensor Network”, 9781-4244-6716-7/10, 2010 IEEE.

[2] Neeta Trivedi, G. Elangovan, S.S. Iyengar, N. Balakrishnan, “A Message-Efficient, Distributed Clustering Algorithm for Wireless Sensor and Actor Networks”, 1-4244-0567-x/06, 2006 IEEE.

[3] Kun Sun, Pai Peng Peng Ning, "Secure Distributed Cluster Formation in Wireless Sensor Networks", Proceedings of the 22nd Annual Computer Security Applications Conference (ACSAC'06), pages 131--140, December 2006.

[4] Vivek Katiyar, Narottam Chand, Surender Soni, "Clustering Algorithms for Heterogeneous Wireless Sensor Network: A Survey”, IJAER, Volume1, No 2, 2010, ISSN-0976-4259

[5] D. J. Dechene, A. El Jardali, M. Luccini, A. Sauer, "A Survey of Clustering Algorithms for wireless Sensor Networks", Information and Automation for Sustainability, 2008. ICIAFS 2008. 4th International Conference, Publication Year: 2008 , Page(s): 295 - 300

[6] G. Gupta, M. Younis, "Load-Balanced Clustering in Wireless Sensor Networks", Pproceedings of International Conference on Communications (ICC 2003), Page(s): 1848 - 1852 , Vol.3 , Anchorage, Alaska, May2003 
International Journal of Wireless \& Mobile Networks (IJWMN) Vol. 4, No. 4, August 2012

[7] M. Xie, Y. Gu, "Multipath Routing Algorithm for Wireless Multimedia Sensor Networks within Expected Network Lifetime", 2010 International Conference on Communication and Mobile Computing 12-14 April 2010,Volume:3 Page(s): 284 - 287, IEEE.

[8] H. Zhang, L. Li, X. Yan, X. Li, “A Load Balancing Clustering Algorithm of WSN for Data Gathering”, 978-1-4577-0536-6/11, 2011, IEEE.

[9] N. Israr \& I. Awan, "Multi-hop clustering Algo. For Load Balancing in WSN", 2006 International Journal of Simulation Vol. 8 No. 1 ISSN 1473-804x

[10] N. Kim, J. Heo, H. S. Kim \& W. H. Kwon, "Reconfiguration of Cluster head for Load Balancing in Wireless Sensor Networks”, Science Direct Computer Communications 31 (2008) 153-159.

[11] A. Akhtar, A. A. Minhas \& S. Jabbaar, "Energy Aware Intra Cluster Routing for Wireless Sensor Networks", International Journal of Hybrid Information Technology, Vol.3, No.1, January, 2010.

[12] E. Laszlo, K. Tornai, G. Treplan \& J. Levendovszky, "Novel Load Balancing Scheduling Algo. For Wireless Sensor Networks", CTRQ 2011 : The Fourth International Conference on Communication Theory, Reliability, and Quality of Service IARIA, 2011.

[13] S. Ozdemir, "Secure Load Balancing via Hierarchical Data Aggregation in Heterogeneous Sensor Networks", Journal of Information Science And Engineering 25, 1691-1705 (2009).

[14] Y. Zhang, Z. Zheng, Y. Jin, X. Wang,” Load Balanced Algorithm In Wireless Sensor Networks Based on Pruning Mechanism”, IEEE transaction, 978-0-7695-3522-7/09.

[15] C. S. Nam, Y. S. Han \& D. R. Shin, Multihop Routing Based on Optimization of the number of cluster heads in Wireless Sensor Networks, Sensors 2011, 11, 2875-2884; doi:10.3390/s110302875.

[16] M.A. Youssef, A. Youssef \& M.F.Younis, "Overlapping Multihop Clustering for WSN”, IEEE Transactions On Parallel And Distributed Systems, Vol. 20, No. 12, December 2009

[17] I.J.Lotf, M.N.Bonab \& S. Khorsandi, “A Novel Cluster Based Routing Protocol with Extending Lifetime for Wireless Sensor Networks”, 2008, IEEE, 978-1-4244-1980-7/08

[18] M. Mahdari, M. Ismail, K. Jumari, "Load Balancing in Energy Efficient Connected Coverage Wireless Sensor Networks", IEEE Transaction, Volume: 02 pp. 448-452, 2009

[19] C. M. Canci, V. Trifa \& A. Martinoli, "Threshold Based Algo. For Power Aware Load Balancing in Sensor Networks", IEEE Transaction, 0-7803-8916-6/05, 2005.

[20] C.P.Low, C. Fang, J. Mee, Ng \& Y.H. Hang, "Load Balanced Clustering Algorithm for Wireless Sensor Networks”, IEEE Communications Society, 1-4244-0353-7/07, 2007.

[21] Ming Ma \& Y. Yang, "Clustering \& Load Balancing in Hybrid Sensor Network with mobile Cluster Heads", Qshine '06', proceedings of the 3rd international conference on quality of service in heterogeneous wired/ wireless Networks, 2006.

[22] Y. Deng, Y. Hu, "A Load balanced Clustering Algorithm for Heterogeneous Wireless Sensor Networks”, 2010, IEEE, pp. 1-4, ISBN: 978-1-4244-7159-1.

[23] J. H. Chang, "An Energy Aware Cluster Based Routing Algorithm for Wireless Sensor Networks", Journal of Information Science \& Engineering, 26, 2159-2171, 2007.

[24] M. Zeynali, L.M.Khanli, A. Mollanejad, "Fuzzy Based Approach for Load Balanced Distributing database on Sensor Networks", International Journal of Future Generation Communication \& Networking, Vol. 3, No. 2, June 2010

[25] M. Iqbal, I. Gondal \& L. Dooley, “An Energy Aware Dynamic Clustering Algorithm for Load Balancing in Wireless Sensor Networks”, Journal Of Communications, Vol. 1, No. 3, June 2006 
International Journal of Wireless \& Mobile Networks (IJWMN) Vol. 4, No. 4, August 2012

[26] Nurhayati, S.H.Choi \& K.O. Lee, “A cluster Based Energy Efficient Location Routing Protocol in Wireless Sensor Networks", International Journal of Computers And Communications, Volume 5, Issue 2, 2011

[27] Bager Zarei1, Mohammad Zeynali and Vahid Majid Nezhad, "Novel Cluster Based Routing Protocol in Wireless Sensor Networks", IJCSI International Journal of Computer Science Issues, Vol. 7, Issue 4, No 1, July 2010.

[28] W. R. Heinzelman, A. Chandrakasan, H. Balakrishnan, "Energy-Efficient Communication Protocol for Wireless Microsensor Networks", Proceedings of the $33^{\text {rd }}$ Annual Hawaill International Conference on System Sciences, Jan 4-7, 2000, Maui. USA. Los Alamitos, CA, USA: IEEE Computer Society, 2000: 223

Dipak W. Wajgi received Bachelor of Engineering degree in Computer Technology from Kavi Kulguru Institute of Technology and Science, Ramtek, Nagpur, India. Presently, he is pursuing his M.Tech. in Computer Science and Engineering from Ramdeobaba College of Engineering and Management, Nagpur, India. He has around twelve years of teaching experience in engineering colleges.

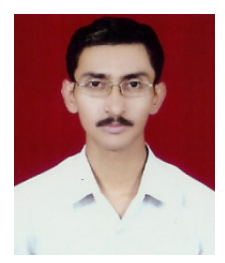

Dr. Nileshsingh V. Thakur received Bachelor of Engineering degree in Computer Science Engineering from Government College of Engineering, Amravati, India and Master of Engineering degree from College of Engineering, Badnera under Amravati University and Sant Gadge Baba Amravati University in 1992 and 2005 respectively. He received Ph.D. degree in Computer Science Engineering under Department of computer Science Engineering from Visvesvaraya National Institute of Technology,

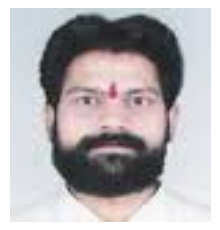
Nagpur, India on $1^{\text {st }}$ February, 2010. His research interest includes image processing, sensor network, computer vision, pattern recognition, artificial neural network and evolutionary approaches. He is having over 20 years of teaching and research experience. Presently, he is Associate Professor in Department of Computer Science and Engineering at Shri Ramdeobaba College of Engineering and Management, Nagpur, India. He is the author or co-author of more than 40 scientific publications in International Journal, International Conferences and National Conferences. Dr. Thakur is a member of editorial board of over eight International journals; also, he is the life member of ISTE, India, IAENG and IAEME. He also worked as the reviewer for international journals and conferences. 